\title{
Vivências de sexualidade na ótica de mulheres na terceira idade
}

\author{
Sexuality experiences by elderly \\ women
}

\section{Rosane dos Santos Polleto Chagas ${ }^{1}$ Fátima Helena Cecchetto ${ }^{2}$ (1) Fernando Riegel ${ }^{3}$ (1)}

\begin{abstract}
1,2Cesuca Centro Universitário (Cachoeirinha). Rio Grande do Sul, Brasil. rosanepolleto@hotmail.com, fatimacecchetto@cesuca.edu.br ${ }^{3}$ Autor para correspondência. Universidade Federal de Mato Grosso (Barra do Garças). Mato Grosso, Brasil. fernandoriegel85@gmail.com
\end{abstract}

RESUMO | OBJETIVO: Compreender as vivências de mulheres na terceira idade em relação à sexualidade. MÉTODO: Revisão integrativa realizada através de cinco etapas descritas por Cooper. A busca foi realizada nas bases de dados LILACS, BDENF e SCIELO, utilizando-se os descritores Saúde da Mulher, Saúde do Idoso, Enfermagem, Terceira Idade, Sexualidade. Foram incluídos 18 artigos. RESULTADOS: Verificou-se nas mulheres da terceira idade a redução ou inexistência de comportamentos relacionados à sexualidade; existência de preconceitos, mitos e tabus sociais e a cultura opressiva; manutenção de práticas sexuais para manter o casamento; constatação de vivências e práticas sexuais inseguras, relacionadas ao não uso do preservativo; compreensão de que a sexualidade envolve aspectos afetivos e emocionais, além do ato sexual. Também foram identificados os fatores positivos e negativos relacionados à vivência da sexualidade pelas mulheres da terceira idade. CONCLUSÃO: Evidenciou-se maior prevalência de fatores negativos relacionados à vivência da sexualidade por estas mulheres do que fatores positivos. São escassos os estudos que constatam a vivência plena da sexualidade e os impactos na qua-lidade de vida da mulher na terceira idade.

DESCRITORES: Saúde da Mulher. Saúde do Idoso. Enfermagem. Terceira Idade. Sexualidade.
ABSTRACT | OBJECTIVE: To understand the experiences of women in old age in relation to sexuality. METHOD: An integrative review carried out through five steps described by Cooper. The search was carried out in the LILACS, BDENF and SCIELO databases, using the descriptors women's health, health of the elderly, nursing, seniors, and sexuality. 18 articles were includ-ing. RESULTS: It was found in the elderly women the reduction or inexistence of behaviors related to sexuality; existence of prejudices, myths and social taboos and oppressive culture; maintaining sexual practices to maintain marriage; verification of unsafe sexual experiences and practices, related to the non-use of condoms; understanding that sexuality involves affec-tive and emotional aspects, in addition to the sexual act. Positive and negative factors related to the experience of sexuality by elderly women were also identified. CONCLUSION: There was a higher prevalence of negative factors related to the experience of sexuality by these women than positive factors. There are few studies that demonstrate the full experience of sexuality and the impacts on the quality of life of women in old age.

DESCRIPTORS: Women's health. Health of the elderly. Nursing. Third age. Sexuality. 


\section{Introdução}

O envelhecimento é um processo de mudanças no organismo humano, não patológico, sequencial e irreversível, de maneira individual e cumulativa. Tratase de uma evolução natural do organismo através de sua deterioração, considerada a última fase do ciclo vital, que envolve afastamento social, perdas psicomotoras e cognitivas, e restrição nos papéis sociais ${ }^{1}$.

Para muitos idosos a velhice está relacionada a aspectos negativos, como a falta de vitalidade, fadiga, doença, isolamento e incapacidade, e é concebida e associada diretamente com a doença, degradação da imagem corporal, perda da autoestima, diminuição do libido, inutilidade, afastamento familiar, e com a morte ${ }^{2}$.

Quando o processo de envelhecimento ocorre de maneira bem-sucedida, a pessoa idosa mostra-se capaz de adaptar-se às novas limitações, mantendose algum grau de satisfação na vida ${ }^{3}$. Dentre outras formas de promoção do envelhecimento ativo e bem sucedido, encontra-se a prática sexual como uma atividade favorável, mesmo em frequência diminuída

A diminuição na frequência de relações sexuais pode estar relacionada ao avanço da idade, uso de medicações, existência de patologias, e diminuição do desejo sexual ${ }^{5}$. Para a mulher idosa, as transformações físicas, hormonais e os padrões religiosos e culturais, as opressões familiares e particularidades individuais consolidam o estigma social de impossibilidade da visão atraente da mesma e sua capacidade de sedução e de vivência plena da sexualidade ${ }^{6}$.

A sexualidade é considerada um conjunto de comportamentos que envolve sentimentos, carícias, palavras, entre outros aspectos, e não somente a relação ou ato sexual propriamente dito ${ }^{3}$. Esta temática extrapola a "questão física ou orgânica, mas tem uma grande influência do aspecto psicológico e de que forma os indivíduos podem exercer sua atividade sexual no decorrer na vida"?.

Entretanto, deve-se considerar que a manutenção ativa da sexualidade na terceira idade tem evidenciado aumento dos casos de doenças sexualmente transmissíveis (DST) e a síndrome da Imunodeficiência Adquirida (AIDS) em muitos casos devido à negligência deste público em relação aos métodos preventivos, além de aspectos relacionados à educação e promoção em saúde ${ }^{7}$.
Diante disso, este estudo justifica-se na necessidade de compreender como as mulheres idosas significam a sexualidade na terceira idade, buscando melhorar a qualidade de vida e prevenir agravos sexualmente transmissíveis, tendo em vista os elevados índices de ISTs nessa etapa da vida. Este estudo teve como questão norteadora: como apresenta-se a sexualidade na ótica de mulheres na terceira idade? O objetivo deste estudo foi compreender as vivências de mulheres na terceira idade em relação à sexualidade.

\section{Método}

Trata-se de uma revisão integrativa que, de acordo com Cooper ${ }^{8}$, deve ser realizada em cinco etapas consecutivas: a formulação do problema, coleta dos dados, avaliação dos dados, análise e interpretação dos dados coletados, e apresentação pública.

Esta pesquisa foi realizada através da busca de artigos científicos na base de dados da Scientific Electronic Library Online (SciELO), da Literatura Latino-Americana e do Caribe em Ciência e Saúde (LILACS), do Banco de Dados da Enfermagem (BDENF), utilizando-se como Descritores em Ciências da Saúde (DECS) para a busca: Saúde da Mulher, Saúde do Idoso, Enfermagem, Terceira Idade, Sexualidade. Foi utilizado o operador booleano "AND" nas seguintes combinações de descritores: saúde da mulher AND sexualidade; Saúde do Idoso AND sexualidade; Saúde da Mulher AND terceira idade. Os critérios de inclusão foram artigos que responderam a questão norteadora, disponíveis nas bases de dados gratuitamente e integralmente em língua portuguesa, publicados nos últimos dez anos (entre 2008 e 2018). Os critérios de exclusão incluíram teses, dissertações, monografias e reportagens jornalísticas.

A coleta de dados ocorreu de março a abril de 2019. Foram identificados 3.216 artigos na busca inicial, sendo 1566 na base de dados LILACS, 871 na BDENF e 776 no banco de dados Scielo. Após a seleção de acordo com os critérios de inclusão, título e duplicidade excluiu-se 3190 artigos, restando 295 artigos, e após a leitura dos resumos foram excluídos 247, por não responder à questão norteadora, restando 48 artigos. Por fim, após proceder a leitura completa dos artigo pré-selecionados, foram incluídos nesta revisão integrativa 18 artigos. 
Figura 1. Fluxograma do processo de seleção dos estudos, referente à busca eletrônica nas bases de dados LILACS, biblioteca SciELO e BDENF , no período compreendido entre os anos de 2008 e 2018 - Cachoeirinha, RS, Brasil, 2020
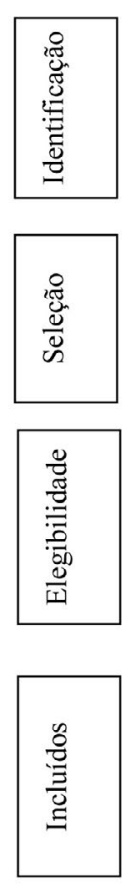

Bases de Dados $(\mathrm{n}=3)$

Artigos Identificados na pesquisa nas bases de dados $=3.216$

Estudos incluídos na amostra Final $=18$
Avaliação por leitura do título e resumo $=295$

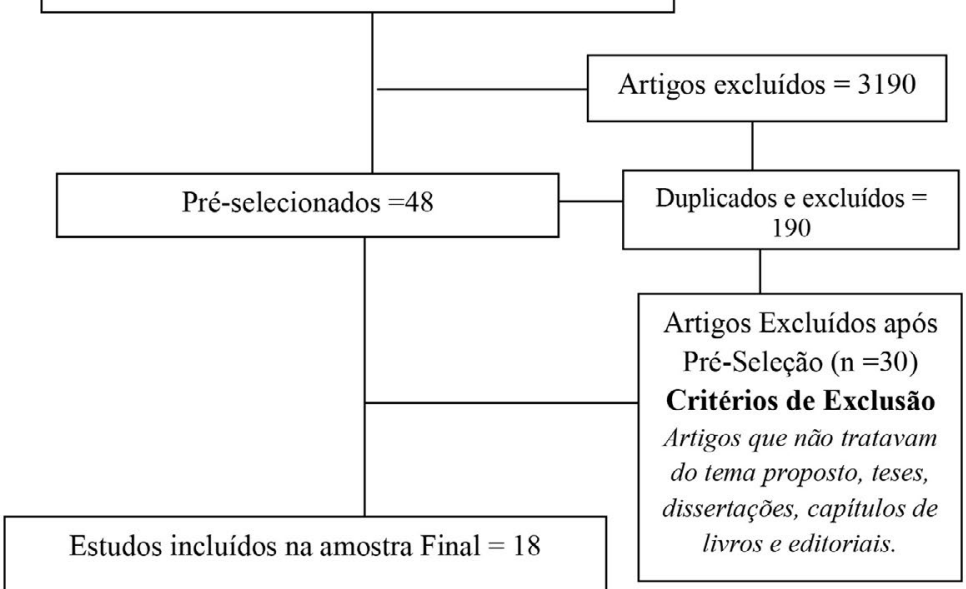

Para executar a avaliação dos dados foi utilizada uma ficha de organização dos dados dos artigos incluídos no estudo, e a sua análise e interpretação ocorreu por meio da sintetização, comparação e discussão dos dados através de quadro sinóptico. Os resultados foram apresentados de forma descritiva através da síntese dos mesmos. Este estudo manteve integralmente os artigos e dados de bases eletrônicos, preservando os autores e respeitando os direitos autorais conforme a Lei ${ }^{\circ} 12.853$, de 14 de agosto de $2013^{9}$.

\section{Resultados}

No Quadro 1 apresenta-se a descrição dos artigos incluídos na presente revisão integrativa. 
Quadro 1. Descrição dos estudos incluídos na revisão integrativa, segundo título e principais resultados. Cachoeirinha, Rio Grande do Sul, Brasil, Brasil, 2019 (continua)

\begin{tabular}{|c|c|c|}
\hline CÓD. AUTORES & $\begin{array}{c}\text { TÍTULO } \\
\end{array}$ & RESULTADOS DAS PESQUISAS \\
\hline $\begin{array}{l}\text { A1 } \\
\text { Arcoverde MAM, Labro- } \\
\text { nici, } \text { LM }^{10}\end{array}$ & $\begin{array}{l}\text { Faces ocultas da sexualidade } \\
\text { emergente de idosos: um estudo } \\
\text { fenomenológico. }\end{array}$ & $\begin{array}{l}\text { - Transcendência da visão biológica do sexo; } \\
\text { - Sexualidade com companheirismo. }\end{array}$ \\
\hline $\begin{array}{l}\text { A2 } \\
\text { Rodrigues DMMR, Labe- } \\
\text { galini CMG, Higarashi IH, } \\
\text { Heidemann ITSB, Baldis- } \\
\text { sera VDA }{ }^{11}\end{array}$ & $\begin{array}{l}\text { O Percurso educativo dialógico } \\
\text { como estratégia de cuidado com } \\
\text { mulheres idosas na sexualidade }\end{array}$ & $\begin{array}{l}\text { - Conceito de sexualidade relacionado ao ato se- } \\
\text { xual; } \\
\text { - Vivência da sexualidade como prática da jovem; } \\
\text { - Existência de preconceitos. }\end{array}$ \\
\hline $\begin{array}{l}\text { A3 } \\
\text { Burigo GF, Fachini } \\
\text { IH, Garetti B, Streicher } \\
\text { CCl, Rosa RS }\end{array}$ & $\begin{array}{l}\text { Sexualidade e comportamento } \\
\text { de idosos vulneráveis a doenças } \\
\text { sexualmente transmissíveis }\end{array}$ & $\begin{array}{l}\text { - Vivências e práticas sexuais inseguras; } \\
\text { - Vulnerabilidade às IST. }\end{array}$ \\
\hline $\begin{array}{l}\text { A4 } \\
\text { Moreira TM, Parreira } \\
\text { BDM, Diniz MA, Silva SR }{ }^{13}\end{array}$ & $\begin{array}{l}\text { Conhecimento das mulheres ido- } \\
\text { sas sobre doenças sexualmente } \\
\text { transmissíveis, conhecimento, } \\
\text { uso e acesso aos métodos pre- } \\
\text { ventivos. }\end{array}$ & $\begin{array}{l}\text { - Conhecimento intermediário acerca das DST e } \\
\text { modos de prevenção, sem hábito do uso de } \\
\text { preservativos. }\end{array}$ \\
\hline $\begin{array}{l}\text { A5 } \\
\text { Marques ADB, Silva RP, } \\
\text { Sousa SS, Santana RS, } \\
\text { Deus SRM; Amorim RF }{ }^{14} \\
\end{array}$ & $\begin{array}{l}\text { A vivência da sexualidade de ido- } \\
\text { sos em um centro de convivên- } \\
\text { cia. }\end{array}$ & $\begin{array}{l}\text { - Sexualidade ativa, além da prática sexual, envol- } \\
\text { vendo sentimentos. }\end{array}$ \\
\hline $\begin{array}{l}\text { A6 } \\
\text { Cunha LM, Mota WS, } \\
\text { Gomes SC, Ribeiro FMA, } \\
\text { Bezerra IMP, Machado } \\
\text { MFAS, Quirino GS }{ }^{15} \\
\end{array}$ & $\begin{array}{l}\text { Vovó e vovô também amam: se- } \\
\text { xualidade na terceira idade }\end{array}$ & $\begin{array}{l}\text { - Difícil abordagem do assunto nas consultas às } \\
\text { idosas. }\end{array}$ \\
\hline $\begin{array}{l}\text { A7 } \\
\text { Frugoli A, Magalhães } \\
\text { JCAO }^{16}\end{array}$ & $\begin{array}{l}\text { A sexualidade na terceira idade } \\
\text { na percepção de um grupo de } \\
\text { idosas e indicações para a edu- } \\
\text { cação sexual. }\end{array}$ & $\begin{array}{l}\text { - Sexualidade e ato sexual como sinônimos; } \\
\text { - } \quad \text { Possibilidade de vida sexual ativa e saudável; } \\
\text { - } \quad \text { Não uso de preservativo após os } 60 \text { anos; } \\
\text { - } \quad \text { Dúvidas, medos e preconceitos sobre o tema; } \\
\text { - Pouca vivência da sexualidade. }\end{array}$ \\
\hline $\begin{array}{l}\text { A8 } \\
\text { Coelho DNP, Daher DV, } \\
\text { Santana RF, Santo FHE }\end{array}$ & $\begin{array}{l}\text { Percepção de mulheres idosas } \\
\text { sobre sexualidade: implicações } \\
\text { de gênero e no cuidado de en- } \\
\text { fermagem }\end{array}$ & $\begin{array}{l}\text { - } \quad \text { Dificuldade em expressar a sexualidade; } \\
\text { - Vivência de conflitos, confundindo sexo, relação } \\
\text { sexual e sexualidade. }\end{array}$ \\
\hline $\begin{array}{l}\text { A9 } \\
\text { Oliveira EL, Neves ALM, } \\
\text { Silva IR }{ }^{17}\end{array}$ & $\begin{array}{l}\text { Sentidos de sexualidade entre } \\
\text { mulheres idosas: relações de gê- } \\
\text { nero, ideologias mecanicistas e } \\
\text { subversão }\end{array}$ & $\begin{array}{l}\text { - Sexo como obrigação; } \\
\text { - Falta de vontade X Existência de vontade de fazer } \\
\text { sexo. }\end{array}$ \\
\hline $\begin{array}{l}\text { A10 } \\
\text { Pereira D, Ponte F, } \\
\text { Costa } E^{18}\end{array}$ & $\begin{array}{l}\text { Preditores das atitudes negativas } \\
\text { face ao envelhecimento e face à } \\
\text { sexualidade na terceira idade }\end{array}$ & $\begin{array}{l}\text { - Presença de atitudes negativas face ao envelheci- } \\
\text { mento e menos permissivas acerca da sexuali- } \\
\text { dade na terceira idade. }\end{array}$ \\
\hline $\begin{array}{l}\text { A11 } \\
\text { Uchôa YS, Costa DCA, } \\
\text { Silva JIAP, Silva STSE, Frei- } \\
\text { tas WMTM, Soares SCS7 }\end{array}$ & $\begin{array}{l}\text { A sexualidade sob o olhar da } \\
\text { pessoa idosa. }\end{array}$ & $\begin{array}{l}\text { - Mito de que idosos são assexuados devido: falta } \\
\text { de informações; alterações fisiológicas e precei- } \\
\text { tos. }\end{array}$ \\
\hline $\begin{array}{l}\text { A12 } \\
\text { Alencar DL, Marques, } \\
\text { APO, Leal MCC, } \\
\text { Vieira JCM }{ }^{19}\end{array}$ & $\begin{array}{l}\text { Exercício da sexualidade em pes- } \\
\text { soas idosas e os fatores relacio- } \\
\text { nados. }\end{array}$ & $\begin{array}{l}\text { - Sexualidade relacionada à genitalidade; } \\
\text { - } \quad \text { Existência de atividade sexual; } \\
\text { - } \quad \text { Prática de autoerotização. }\end{array}$ \\
\hline $\begin{array}{l}\text { A13 } \\
\text { Queiroz MAC, Lourenço } \\
\text { RME, Coelho MMF, Mi- } \\
\text { randa KCL, Barbosa RGB, } \\
\text { Bezerra STF20 }\end{array}$ & $\begin{array}{l}\text { Representações sociais da sexu- } \\
\text { alidade entre idosos. }\end{array}$ & $\begin{array}{l}\text { - Amor, respeito e carinho são elementos centrais } \\
\text { da sexualidade na terceira idade. }\end{array}$ \\
\hline $\begin{array}{l}\text { A14 } \\
\text { Maschio MBM, Balbino } \\
\text { AP, Souza PFR, Kalinke } \\
\text { LP21 }\end{array}$ & $\begin{array}{l}\text { Sexualidade na terceira } \\
\text { idade: medidas de prevenção } \\
\text { para doenças sexualmente } \\
\text { transmissíveis e AIDS }\end{array}$ & $\begin{array}{l}\text { Os idosos possuem desejos, necessidades sexu- } \\
\text { ais e fazem projetos para o futuro. }\end{array}$ \\
\hline
\end{tabular}


Quadro 1. Descrição dos estudos incluídos na revisão integrativa, segundo título e principais resultados. Cachoeirinha, Rio Grande do Sul, Brasil, Brasil, 2019 (conclusão)

\begin{tabular}{|c|c|c|}
\hline CÓD. AUTORES & TÍTULO & RESULTADOS DAS PESQUISAS \\
\hline $\begin{array}{l}\text { A15 } \\
\text { Moraes KM, Vasconcelos } \\
\text { DP, Silva ASR, Silva RCC, } \\
\text { Santiago LMM, Freitas } \\
\text { CASL }^{22}\end{array}$ & $\begin{array}{l}\text { Companheirismo e sexualidade } \\
\text { de casais na melhor idade: cui- } \\
\text { dando do casal idoso }\end{array}$ & $\begin{array}{l}\text { - Sentimentos envolvendo a sexualidade; } \\
\text { - Há diminuição no padrão da atividade sexual; } \\
\text { - Preconceito quanto à manifestação de carinho; } \\
\text { - Fortalecimento do vínculo. }\end{array}$ \\
\hline $\begin{array}{l}\text { A16 } \\
\text { Souza M, Marcon SS, } \\
\text { Bueno SMV, Carreira L, } \\
\text { BaldisserA VDA }\end{array}$ & $\begin{array}{l}\text { A vivência da sexualidade por } \\
\text { idosas viúvas e suas percepções } \\
\text { quanto à opinião dos familiares } \\
\text { a respeito }\end{array}$ & $\begin{array}{l}\text { - Vivência da sexualidade de forma não plena; } \\
\text { - Opressão da sociedade e da família; } \\
\text { - Falta de incentivo dos familiares. }\end{array}$ \\
\hline $\begin{array}{l}\text { A17 } \\
\text { Biasus F, Demantova A, } \\
\text { Camargo BV }{ }^{24}\end{array}$ & $\begin{array}{l}\text { Representações sociais do enve- } \\
\text { lhecimento e da sexualidade } \\
\text { para pessoas com mais de } 50 \\
\text { anos. }\end{array}$ & $\begin{array}{l}\text { - Sexualidade enquanto sentimentos; } \\
\text { - Menor frequência nas relações sexuais, mas } \\
\text { maior qualidade, devido a problemas de saúde; } \\
\text { - Falta de parceiro como barreira para sexualidade }\end{array}$ \\
\hline $\begin{array}{l}\text { A18 } \\
\text { Oliveira DC, Cotica } \mathrm{CS}^{25}\end{array}$ & $\begin{array}{l}\text { Sexualidade e qualidade de vida } \\
\text { na idade avançada. }\end{array}$ & $\begin{array}{l}\text { - Diminuição da atividade e desejo sexual devido à } \\
\text { idade, patologias, uso de medicamentos; } \\
\text { - Sexualidade revelada através de sentimentos, ul- } \\
\text { trapassando o ato sexual. }\end{array}$ \\
\hline
\end{tabular}

Fonte: Os autores (2019).

Ao realizar a técnica de análise de conteúdo dos artigos encontrados e incluídos no estudo, foi possível verificar 10 eixos apontados pelas mulheres da terceira idade como determinantes e relacionados à vivência da sexualidade pelas mesmas. Também foram identificados os fatores positivos e negativos relacionads à vivência da sexualidade pelas mulheres da terceira idade, apresentados no quadro 2.

Quadro 2. Fatores relacionados à vivência da sexualidade pelas mulheres da terceira idade. Cachoeirinha, Rio Grande do Sul, Brasil, Brasil, 2019

\begin{tabular}{|c|c|}
\hline Fatores positivos & Fatores negativos \\
\hline $\begin{array}{l}\text { - Manutenção da sexualidade ativa; } \\
\text { - Associação da sexualidade com envolvi- } \\
\text { mento afetivo e emocional. }\end{array}$ & $\begin{array}{l}\text {-Diminuição da vivência da sexualidade na terceira idade; } \\
\text {-Associação restrita da sexualidade ao ato sexual; } \\
\text {-Existência de preconceitos em relação à vivência da sexualidade e o } \\
\text { envelhecimento pela idosa e o mito de que idosos são assexuados; } \\
\text {-Vivências e práticas sexuais inseguras, especialmente relacionadas à } \\
\text { má adesão ao uso do preservativo, tornando a mulher mais vulnerável } \\
\text { a infecções sexualmente transmissíveis; } \\
\text {-Continuidade de práticas sexuais devido às obrigações do casamento } \\
\text { em detrimento do desejo; } \\
\text {-Cultura opressiva da sociedade e da família em relação à vivência da } \\
\text { sexualidade de idosas. }\end{array}$ \\
\hline
\end{tabular}

Fonte: Os autores (2019). 
Dos fatores levantados na pesquisa, verificou-se a prevalência de fatores negativos relacionados à vivência da sexualidade pelas mulheres idosas, o que está muito atrelato à cultura e ao preconceito, tendo a sociedade atribuído sexualidade ao ato sexual propriamente dito, o que com a avançada idade fica comprometido, porém, qualquer forma de exercitar a sexualidade deve ser valorizada e introduzida no cotidiano singular das mulheres idosas com o intuito de preservar sua natureza humana e a sua privacidade.

O fato de viver a sexualidade na terceira idade não pode ser motivo de constrangimento pelas idosas, pelo contrário, deve-se levar em consideração que essa fase da vida leva à diminuição da libido e dos hormônios sexuais femininos, o que pode causar diminuição do desejo sexual e não limitar os seus desejos e vontade em relação à sexualidade.

\section{Discussão}

A redução das vivências de sexualidade na terceira idade foi citada em nove estudos ( $A 2, A 7, A 8, A 9, A 11$, A15, A16, A17 e A18). Ribeiro, Alves e Meira ${ }^{26}$ associam fatores relacionados ao sistema reprodutor da mulher na terceira idade com a diminuição no padrão da atividade sexual, justificado pelo climatério, decorrente da falência da função ovariana, com diminuição da produção de esteroides e ausência de ovulação, bem como a redução da libido é atribuída, entre outros fatores, à atrofia vaginal, perda das rugosidades e redução da secreção vaginal, causando dispareunia e diminuição na sensação de prazer.

Alencar et al. $\stackrel{27}{ }$ acrescentam que as mudanças corporais e psicológicas da idosa podem gerar insatisfação com a própria imagem corporal, refletindo na não aceitação de si própria, gerando a recusa para a vivência da sexualidade.

Dos estudos avaliados, quatro ( $A 2, A 7, A 8, A 9$ e $A 12)$ referiram que as mulheres da terceira idade associam a sexualidade com o ato sexual em si, ou seja, o coito. Bastos et al. $\stackrel{28}{ }$ e Vieira, Coutinho e Saraiva ${ }^{1}$ associam este achado devido sua característica de função fisiológica, deixando de considerar outros meios de expressão da sexualidade, tais como manifestações de afeto, carinho, ternura e companheirismo.
A existência de preconceitos quanto à vivência da sexualidade associada ao envelhecimento pelas próprias idosas, suas famílias e sociedade foi apresentado por nove estudos (A2, A6, A7, A8, A9, A10, A11, A15 e A16). Neste sentido, Almeida e Lourenço ${ }^{29}$ relacionaram esses preconceitos aos estereótipos de aparência física não atraente, falta de interesse e sensibilidade aos estímulos sexuais, bem como a falta de informação, que conduzem às atitudes pessimistas.

A sociedade categoriza esse período da vida como assexual, existindo o preconceito pela própria idosa e sua família, que não tolera a manutenção de relações amorosas entre seus idosos, sendo os filhos os primeiros a negarem a sexualidade dos pais, associando a sexualida-de a um sinal de demência ${ }^{30}$.

As vivências e práticas sexuais inseguras, especialmente relacionadas ao não uso do preservativo, tornando a mulher mais vulnerável a doenças sexualmente transmissíveis foi apresentado em três estudos (A3, A4 e A7).

A baixa adesão ao uso do preservativo pode estar relacionada ao fato da falta deste dispositivo de prevenção no ato da prática sexual dos idosos, ou a falta de destreza no uso, causando lentidão, o que na visão do idoso pode atrapalhar a ocasião íntima. Especialmente em relação às mulheres na terceira idade, os argumentos estão relacionados com a despreocupação com a anticoncepção, dificuldade de negociação entre os parceiros, bem como a confi-ança estabelecida entre o casamento e o relacionamento estável, presumindo que podem abrir mão do preservativo nas relações sexuais, gerando maior vulnerabilidade para infecções por DSTs/HIV31-33.

Dornelas Neto et al. ${ }^{34}$ acrescentam que o aumento à exposição a situações de risco devido aos casos de divórcio, a viuvez, a busca de parceiros sexuais nas redes sociais e sites da internet e aumento do turismo/ comércio sexual, bem como as alterações fisiológicas decorrentes do processo de envelhecimento podem contribuir para o aumento do risco de vulnerabili-dade às DST/HIV.

Cezar, Aires e Paz ${ }^{35}$ ressalta a importância do desenvolvimento de programas de saúde pública voltados especificamente aos idosos, de forma a esclarecer dúvidas relacionadas à sexualidade e à prevenção de 
DSTs, permitindo aos mesmos adotar práticas mais saudáveis e seguras relacionadas à vivência da sexualidade, principalmente com o cenário onde há o aumento da expectativa de vida ${ }^{\underline{36}}$.

A continuidade de práticas sexuais concebida como uma obrigação do casamento e o não por desejo foi apontado por dois estudos (A9, A12). Ferreira et al. ${ }^{37}$ revelou que as mulheres mantém relações sexuais devido sua obrigação enquanto esposa/companheira e possuem o entendimento de que se não cumprirem com a sua obrigação, o marido/companheiro irá buscar essa satisfação em relações ou práticas sexuais fora do casamento, assim como Berger 38 relata que as idosas justificam a importância da relação sexual para manter o parceiro.

A existência da cultura opressiva da sociedade e da família com relação à vivência da sexualidade nas mulheres da terceira idade foi apontada por quatro estudos (A7, A10, A11 e A16). Baldissera e Bueno ${ }^{39}$ revelam que tiveram dificuldades na abordagem do tema sexualidade, pois na fala das mulheres idosas existem tabus e o mitos, bem como a representação social de não falar sobre sexo, enquanto prática socialmente construída.

A maior parte das idosas tem vergonha em assumir que possuem vida sexual ativa, principalmente porque a família e a sociedade as desencorajam, e a mídia estampa a vivência da sexualidade por idosos como pejorativa ${ }^{40}$.

Em relação aos fatores positivos para a vivência da sexualidade, a manutenção da sexualidade ativa pelas mulheres da terceira idade é apontada como aspecto positivo para a vivência da sexualidade por quatro estudos (A5, A7, A12, A14). Esta valorização também é apresentada por Correia et al. ${ }^{41}$ que relacionam o bem-estar geral com a saúde sexual satisfatória, e a considera um fator importante e fundamental para a qualidade de vida. As idosas reconhecem que a vivência da sexuallidade proporciona bem-estar físico e psicológico, e contribui para a diminuição de problemas físicos e de saúde mental associados com o envelhecimento ${ }^{42}$.
A vivência da sexualidade associada ao envolvimento afetivo e emocional foi apresentada por seis estudos (A1, A5, A13, A15, A17, A18). Vieira, Coutinho e Saraiva ${ }^{1}$ denota que esta vivência de prazer só será atingida se houver afetividade na relação, envolvendo carícias, toque, cumplicidade, sentimentos e intimidade durante a relação sexual, indo além do simples ato.

A sexualidade não está restrita à relação sexual, mas compreende a interação entre a afetividade, a genitalidade, a relação interpessoal, um conjunto de comportamentos e sentimentos, exigindo contato, intimidade e carinho, sendo uma linguagem e meio de expressão do ser humano, essencial para o bem estar físico e emocional dos idosos ${ }^{42}$.

\section{Conclusão}

Com este estudo foi possível evidenciar que a redução ou inexistência de momentos envolvendo a sexualidade entre as idosas e as causas podem estar relacionadas principalmente à associação ao ato sexual, relacionadas à inaptidão física, a alteração na imagem corporal e as patologias desenvolvidas no processo do envelhecimento. A existência de preconceitos, mitos e tabus sociais quanto a vivência da sexualidade e o conceito de assexualidade das idosas impedem a plenitude da sexualidade, assim como a cultura opressiva existente na sociedade.

Um fator preocupante foi a constatação de vivências e práticas sexuais inseguras, especialmente relacionadas ao não uso do preservativo, tornando a muIher idosa mais vulnerável a doenças sexualmente transmissíveis. Das limitações do estudo destaca-se a escassez de pesquisas realizadas em nível nacional com foco na sexualidade da pessoa idosa.

Entretanto, estratégias voltadas para a promoção e prevenção da saúde da mulher idosa devem abordar essa temática, objetivando a compreensão pelas muIheres idosas de que a sexualidade envolve mais do que o simples ato sexual, mas também afeto e emoções, tornando sua vivência mais saudável frente aos benefícios físicos e psicológicos proporcionados, o que pode refletir diretamente na qualidade de vida da mulher na terceira idade. 


\section{Contribuições dos autores}

Chagas RSP participou da concepção, delineamento, busca e análise estatística dos dados da pesquisa, interpretação dos resultados, redação do artigo científico. Cecchetto $\mathrm{FH}$ participou da orientação, coleta de dados da pesquisa, interpretação dos dados e redação. Riegel F participou da concepção, delineamento, interpretação dos resultados e redação do artigo científico.

\section{Conflitos de interesses}

Nenhum conflito financeiro, legal ou político envolvendo terceiros (governo, empresas e fun-dações privadas, etc.) foi declarado para nenhum aspecto do trabalho submetido (incluindo, mas não se limitando a subvenções e financiamentos, participação em conselho consultivo, desenho de estudo, preparação de manuscrito, análise estatística, etc.).

\section{Referências}

1. Vieira KFL, Coutinho MPL, Saraiva ERA. A Sexualidade na Velhice: representações sociais de Idosos frequentadores de um Grupo de Convivência. Psicol Ciênc Prof. 2016;36(1):196-209. http://dx.doi.org/10.1590/1982-3703002392013

2. Ploner KS, Michels LRF, Oliveira MAM, Strey MN. O significado de envelhecer para homens e mulheres. Cidadania e participação social. [Internet]. 2018:142-158. Disponível em: http://books. scielo.org/id/hn3q6/pdf/silveira-9788599662885-14.pdf

3. Coelho DNP, Daher DV, Santana RF, Santo FHE . Percepção de mulheres idosas sobre sexualidade: implicações de gênero e no Cuidado de enfermagem [Internet]. Rev RENE. 2010;11(4):163173. Disponível em: http://www.periodicos.ufc.br/rene/article/ view/4641

4. Debert $G$, Brigeiro $M$. Fronteiras de gênero e a sexualidade na velhice. RBCS. 2012;27(80):37-54. http://dx.doi.org/10.1590/S0102$\underline{69092012000300003}$

5. Moraes KM, Vasconcelos DP, Silva ASR, Silva RCC, Santiago LMM, Freitas CASL. Companheirismo e sexualidade de casais na melhor idade: cuidando do casal idoso. Rev Bras Geriatr Gerontol. 2011; 14(4):787-798. http://dx.doi.org/10.1590/S180998232011000400018

6. Souza M, Marcon SS, Bueno SMV, Carreira L, Baldissera VDA. A vivência da sexualidade por idosas viúvas e suas percepções quanto à opinião dos familiares a respeito. Saúde Soc. 2015;24(3):936-944. http://dx.doi.org/10.1590/S010412902015132060

7. Uchôa YS, Costa DCA, Silva Júnior IAP, Silva STSE, Freitas WMTM, Soares SCS. A sexualidade sob o olhar da pessoa idosa. Revista brasileira de geriatria e gerontologia 2016;19(6):939-949. http:// dx.doi.org/10.1590/1981-22562016019.150189
8. Cooper HM. The integrative research review: a systematic approach. Beverly Hills: Sage; 1989.

9. Brasil. Presidência da República. Lei n 9.610, de 19 de fevereiro de 1998: Altera, atualiza e consolida a legislação sobre direitos autorais e dá outras providências. [Internet]. Brasília, DF; 1998. Disponível em: http://www.planalto.gov.br/ccivil_03/leis/l9610.htm

10. Arcoverde MAM, Labronici LM. Faces ocultas da sexualidade emergente de idosos: um estudo fenomenológico. Rev Bras Enferm. 2008;7(3):1-6. http://dx.doi.org/10.5935/16764285.20081934

11. Rodrigues DMMR, Labegalini CMG, Higarashi IH, Heidemann ITSB, Baldissera VDA. O Percurso educativo dialógico como estratégia de cuidado com mulheres idosas na sexualidade. Esc Anna Nery. 2018;22(3):e20170388. http://dx.doi.org/10.1590/21779465-ean-2017-0388

12. Burigo GF, Fachini IH, Garetti B, Streicher CCl, Rosa RMS. Sexualidade e Comportamento de Idosos Vulneráveis a Doenças Sexualmente Transmissíveis. CuidArte Enfermagem [Internet]. 2015;9(2):148-153. Disponível em: https://pesquisa.bvsalud.org/ portal/resource/pt/bde-27676

13. Moreira TM, Parreira BDM, Diniz MA, Silva SR. Conhecimento das mulheres idosas sobre doenças sexualmente transmissíveis, conhecimento, uso e acesso aos métodos preventivos. Rev Eletr Enfer. 2012;14(4):803-810. http://dx.doi.org/10.5216/ree. v14i4.13766

14. Marques ADB, Silva RP, Sousa SS, Santana RS, Deus SRM, Amorim RF. A vivência da sexualidade de idosos em um centro de convivência. R Enferm Cent O Min. 2015; 5(3):1768-1783. http:// dx.doi.org/10.19175/recom.v5i3.913

15. Cunha LM, Mota WS, Gomes SC, Ribeiro Filho MA, Bezerra IMP, Machado MFAS et al. Vovó e vovô também amam: sexualidade na terceira idade. Rev Min Enferm. 2015;19(4):901-906. http://dx.doi. org/10.5935/1415-2762.20150069

16. Frugoli A, Magalhães Junior CAO. A sexualidade na terceira idade na percepção de um grupo de idosas e indicações para a educação sexual. Arq Ciênc Saúde. 2011;15(1):1-10. http://dx.doi. org/10.25110/arqsaude.v15i1.2011.3696

17. Oliveira EL, Neves ALM, Silva IR. Sentidos de sexualidade entre mulheres idosas: relações de gênero, ideologias mecanicistas e subversão. Psicol Soc. 2018;30(1):e166019. http://dx.doi. org/10.1590/1807-0310/2018v30166019

18. Pereira D, Ponte F, Costa E. Preditores das atitudes negativas face ao envelhecimento e face à sexualidade na terceira idade. Aná Psicológica. 2018;36(1):31-46. http://dx.doi.org/10.14417/ ap.1341

19. Alencar DL, Marques APO, Leal MCC, Vieira JCM. Exercício da sexualidade em pessoas idosas e os fatores relacionados. Rev Bras Geriatr Gerontol. 2016;19(5):861-869. http://dx.doi. org/10.1590/1809-98232016019.160028 
20. Queiroz MAC, Lourenço RME, Coelho MMF, Miranda KCL, Barbosa RGB, Bezerra STF. Representações sociais da sexualidade entre idosos. Rev Bras Enferm. 2015;68(4):662-667. http://dx.doi. org/10.1590/0034-7167.2015680413i

21. Maschio MBM, Balbino AP, Souza PFR, Kalinke LP. Sexualidade na terceira idade: medidas de prevenção para doenças sexualmente transmissíveis e AIDS. Rev Gaúcha de Enferm. 2011;32(3):583-9. http://dx.doi.org/10.1590/S198314472011000300021

22. Moraes KM, Vasconcelos DP, Silva ASR, Silva RCC, Santiago LMM, Freitas CASL. Companheirismo e sexualidade de casais na melhor idade: cuidando do casal idoso. Rev Bras Geriatr Gerontol. 2011;14(4):787-798.

23. Souza M, Marcon SS, Bueno SMV, Carreira L, Baldissera VDA. A vivência da sexualidade por ido-sas viúvas e suas percepções quanto à opinião dos familiares a respeito. Saúde Soc. 2015;24(3):936-944. http://dx.doi.org/10.1590/S010412902015132060

24. Biasus F, Demantova A, Camargo BV. Representações sociais do envelhecimento e da sexualidade para pessoas com mais de 50 anos. Temas Psicol. [Internet]. 2011;19(1):319-336. Disponível em: http://pepsic.bvsalud.org/pdf/tp/v19n1/v19n1a25.pdf

25. Oliveira DC, Cotica CS. Sexualidade e qualidade de vida na idade avançada. Geriatria \& Gerontologia [Internet]. 2009;3(1):4148. Disponível em: http://ggaging.com/details/318/pt-BR

26. Ribeiro LCC, Alves PB, Meira EP. Percepção dos idosos sobre as alterações fisiológicas do envelhecimento. Ciênc Cuid Saúde. 2009;8(2):220-227. http://dx.doi.org/10.4025/cienccuidsaude. v8i2.8202

27. Alencar DL, Marques APO, Leal MCC, Vieira JCM. Fatores que interferem na sexualidade de idosos: uma revisão integrativa. Ciênc Saúde Coletiva 2014; 19(8):3533-3542. http://dx.doi. org/10.1590/1413-81232014198.12092013

28. Bastos CC, Closs VE, Pereira AMVB, Batista C, Idalêncio FA, Carli GA et al. Importância atribuída ao sexo por idosos do município de Porto Alegre e associação com a autopercepção de saúde e o sentimento de felicidade. Rev Bras Geriatr Gerontol. 2012;15(1):87-95. http://dx.doi.org/10.1590/S1809$\underline{98232012000100010}$

29. Almeida T, Lourenço ML. Amor e sexualidade na velhice: direito nem sempre respeitado. RBCEH. 2008;5(1):130-140. http:// dx.doi.org/10.5335/rbceh.2012.104

30. Araújo ACF. Rompendo o silêncio: desvelando a sexualidade em idosos. Revista UNILUS Ensino e Pesquisa [Internet]. 2015;12(29):35-41. Disponível em: http://revista.unilus.edu.br/ index.php/ruep/article/view/689
31. Laroque MF, Affeldt ÂB, Cardoso DH, Souza GL, Santana MG, Lange $C$. Sexualidade do idoso: comportamento para a prevenção de DST/AIDS. Rev Gaúcha Enferm. 2011;32(4):774-80. http:// dx.doi.org/10.1590/S1983-14472011000400019

32. Andrade J, Ayres JA, Alencar RA, Duarte MTC, Parada CMGL. Vulnerabilidade de idosos a infec-ções sexualmente transmissíveis. Acta Paul Enferm. 2017;30(1):8-15. http://dx.doi. org/10.1590/1982-0194201700003

33.Pedrosa NL, Paiva SS, Almeida LRF, Holanda ER, Kerr LRFS, Galvão MTG. The historic data series on AIDS in the state of Ceará, Brazil. Ciênc Saúde Colet. 2015;20(4):1177-84. http://dx.doi. org/10.1590/1413-81232015204.00582014

34.Dornelas Neto J, Nakamura AS, Cortez LER, Yamaguchi MU. Doenças sexualmente transmissíveis em idosos: uma revisão sistemática. Ciência \& Saúde Coletiva. 2015;20(12):3853-3864. http://dx.doi.org/10.1590/1413-812320152012.17602014

35. Cezar AK, Aires M, Paz AA. Prevenção de doenças sexualmente transmissíveis na visão de ido-sos de uma Estratégia da Saúde da Família. Rev Bras Enferm. 2012;65(5):745-50. http://dx.doi. org/10.1590/S0034-71672012000500005

36. Hughes AK, Rostant OS, Pelon S. Sexual problems among older women by age and race. J Wo-mens Health. 2015;24(1):6639. http://dx.doi.org/ 10.1089/jwh.2014.5010

37. Ferreira KS, Silva MG, Cherem TMDA, Araújo CLO. Percepção dos idosos perante o sexo na ida-de avançada. Geriatria \& Gerontologia [Internet]. 2009;3(4):182-188. Disponível em: http:// www.ggaging.com/details/290/pt-BR/percepcao-dos-idososperante-o-sexo-na-idade-avancada

38. Berger M. “Amor sem sexo é amizade. Sexo sem amor é vontade": vida sexual na terceira idade. Revista Kairós Gerontologia 2012; 15(8):127-154. https://doi.org/10.23925/2176901X.2012v15i4p127-154

39. Baldissera VDA, Bueno SMV. A representação da sexualidade por idosas e a educação para a saúde. Rev Eletr Enf. 2010;12(4):622-9. http://dx.doi.org/10.5216/ree.v12i4.8830

40. Bortolotti MC, Brutscher IS, Kist VF, Bard PF, Bavaresco AM. A Sexualidade em idosos. Psicologado. 2013;10(1):22-31.

41. Correia LS, Brasil C, Silva MD, Silva DFC, Amorim HO, Lordêlo P. Função sexual e qualidade de vida de mulheres: um estudo observacional. Rev Port Med Geral Fam. [Internet]. 2016;32(6):405-9. Disponível em: http://www.scielo.mec.pt/pdf/ rpmgf/v32n6/v32n6a07.pdf

42. Oliveira LB, Baía RV, Delgado ART, Vieira KFL, Lucena ALR. Sexualidade e envelhecimento: avaliação do perfil sexual de idosos não institucionalizados. Rev Ciênc Saúde Nova Esperança. 2015; 13(2):42-50. http://dx.doi.org/10.17695/ revcsnevol13n2p42\%20-\%2050 